\title{
Oral Hygiene and Dentition Status in Patients with Congenital Hemorrhagic Disorders: A Comparative Study
}

\author{
Mathangi Kumar ${ }^{1}$, Keerthilatha M. Pai ${ }^{1}$, Ravindranath Vineetha ${ }^{1}$, Annamma Kurien²®
}

\begin{abstract}
${ }^{1}$ Department of Oral Medicine and Radiology, Manipal College of Dental Sciences, Manipal, Manipal Academy of Higher Education, Manipal, Karnataka, India.

2Department of Pathology, Melaka Manipal Medical College, Manipal Academy of Higher Education, Manipal, Karnataka, India.
\end{abstract}

Author to whom correspondence should be addressed: Keerthilatha M. Pai, Department of Oral Medicine and Radiology, Manipal College of Dental Sciences, Manipal, Manipal Academy of Higher Education, Manipal, 576104, Karnataka, India. Phone: 0820 2922455. E-mail: keerthilatha.pai@manipal.edu.

Academic Editors: Alessandro Leite Cavalcanti and Wilton Wilney Nascimento Padilha

Received: 15 November 2019 / Accepted: 30 March 2020 / Published: 03 April 2020

How to cite this article: Kumar M, Pai KM, Vineetha R, Kurien A. Oral hygiene and dentition status in patients with congenital hemorrhagic disorders: a comparative study. Pesqui Bras Odontopediatria Clín Integr. 2020; $20: e 5392$. https://doi.org/10.1590/pboci.2020.060

\begin{abstract}
Objective: To compare the status of oral hygiene and dentition in patients with congenital hemorrhagic disorders with their age-matched healthy counterparts. Also, the prevalence of fear of dentists/ dental treatment among these patients was assessed. Material and Methods: This study was performed on children and adults with von Willebrand disease (vWD), hemophilia (A or B), and healthy subjects. Oral health and dentition status was assessed using the simplified oral hygiene index (OHI-S), plaque index, and the decayed, missing, filled teeth (dmft/DMFT) index. One-way ANOVA test was employed to compare the oral hygiene and dentition status of subjects in the three groups. A p-value of $<0.05$ was considered statistically significant. Results: The DMFT score did not vary significantly between the groups ( $>0.05)$. Higher OHI-S scores and a poor oral hygiene status was observed more in the hemophilia group than the vWD group and healthy controls. A total of $27.3 \%$ of the subjects in the vWD group, $18.2 \%$ of subjects in the hemophilia group, and no subjects in the healthy group had a fear of dentists or dental treatment. Conclusion: Subjects with vWD had a higher number of carious teeth when compared to the other groups. Poor oral hygiene status was observed in subjects with hemophilia.
\end{abstract}

Keywords: Blood Coagulation Disorders; von Willebrand Diseases; Hemophilia A; Oral Hygiene. 


\section{Introduction}

Individuals with congenital hemorrhagic disorders constitute a small but significant proportion of the population. Hemophilia and von Willebrand disease are the most common inherited bleeding disorders. Hemophilia is the most common severe bleeding disorder that is inherited in an X-linked recessive manner, which is caused by an absence or reduced activity of factor VIII (hemophilia A) or factor IX (hemophilia B) [1]. von Willebrand disease (vWD) is the second most common congenital bleeding disorder that is inherited either as an autosomal dominant or recessive trait. As a result of either a quantitative and/ or qualitative defect in the Von Willebrand Factor (VWF), normal hemostasis is affected [2].

The prevalence of hemophilia in India is 1 in 5000 for Hemophilia A and 1 in 30,000 for Hemophilia B [1]. von Willebrand disease accounts for only $8.5 \%$ of all inherited bleeding disorders among the Indian population, which is much less common as compared to the Western world [2]. The possible reason for this could be the lack of awareness about the disease, diversity in the laboratory techniques for diagnosing vWD or due to the unavailability of diagnostic amenities required for identifying the disorder [3].

Patients with bleeding disorders experience profuse bleeding even with a trivial trauma, which usually needs plasma/ factor transfusions. They may have excessive bleeding after dental procedures, particularly after tooth extraction. One of the known barriers to providing professional dental care is the lack of confidence among dentists to manage these patients. Since minor trauma from tooth brushing may initiate a bleeding episode and the inherent fear to undergo dental treatment procedures, oral hygiene maintenance and oral health care are often neglected by patients with congenital bleeding disorders [4,5].

Standard methods for the management of intraoral bleeding include oral tranexamic acid, epsilon aminocaproic acid tablets, subcutaneous desmopressin, and local measures like compression with moistened gauze, collagen, hemostatic sponge with sutures and oxidized regenerated cellulose [4,5].

It was observed that individuals with hemophilia had higher debris and calculus scores when compared to their age-matched healthy counterparts, which were indicative of poor orodental status [6]. There is only one study in the literature, which has evaluated the oral health and dentition status of children with von Willebrand disease [7]. Further, the only comparative studies on the oral hygiene and dentition status in children with bleeding disorders and healthy children were performed by previous authors [8,9].

Hence, this study was performed to compare the status of oral hygiene and dentition in children as well as adults with congenital hemorrhagic disorders with their age-matched healthy counterparts. We also assessed the prevalence of fear of dentists/ dental treatment among these patients.

\section{Material and Methods}

Study Design and Sample

This cross-sectional study was conducted in the Department of Oral Medicine, Manipal Academy of Higher Education, in collaboration with the Hemophilia Society, Manipal, India. Patients attending Hemophilia society were included in the study.

Inclusion criteria for the case group were patients diagnosed with von Willebrand disease, hemophilia (A or B) from the Hemophilia Society and inclusion criteria for the control group were age, and gendermatched healthy volunteers with no von Willebrand disease, hemophilia or any other systemic comorbidities who were recruited from the Oral Diagnosis Clinic. Exclusion criteria for both the groups were patients with any other bleeding disorders other than von Willebrand disease, hemophilia and who had other systemic comorbidities. 
Information pertaining to the disease and details regarding oral complaints and oral hygiene practices were collected from each subject and recorded in a specially designed Proforma. The subjects were categorized into three groups: Group 1: von Willebrand group (vWD), Group 2: Hemophilic group and Group 3: Healthy group.

\section{Data Collection}

Clinical examination was carried out in a well-ventilated area with proper illumination. Atraumatic oral examination for the study subjects was performed with blunt-tipped explorers in order to avoid injury to the soft tissues.

The following parameters were recorded: 1) Overall oral hygiene status (Plaque index for children between 2-6 years of age and Simplified Oral Hygiene Index - OHI-S, for adults); 2) Presence or absence of gingival bleeding; 3) Dentition status: DMFT index for adults and dmft index for children; and 4) Presence or absence of fear of dental treatment

Gingiva was examined gently for the presence of bleeding. Whether the bleeding occurred spontaneously or on provocation was noted. The overall dentition status was assessed using the $\mathrm{dmft} / \mathrm{DMFT}$ indices [10]. The dmft index was evaluated in children before the age of exfoliation, while the DMFT index was applied to mixed and permanent dentition. Third molars, teeth extracted for reasons other than dental caries; teeth restored for cosmetic purposes were not included for scoring. To assess the dental fear, the study subjects were asked if they had fear to undergo dental treatment procedures. Also, the reason for fear was noted in the patient's own words.

After clinical examination, all the study subjects were educated regarding the importance of maintaining good oral health. Oral hygiene instructions were given and they were motivated to get regular oral health checkups in order to prevent invasive dental treatment in the future.

\section{Statistical Analysis}

The data obtained from all the subjects were entered in Microsoft Excel spreadsheet and the statistical analysis was performed using SPSS version 21.0 (IBM SPSS Corp., Armonk, NY, USA). One-way ANOVA test was employed to compare the oral hygiene and dentition status of subjects in the three groups. A p-value of $<0.05$ was considered statistically significant.

\section{Ethical Aspects}

Institutional Ethical Committee approval was obtained before conducting the study (IEC 549/2014). All the subjects were informed regarding the study and were provided with the subject information sheet. Written informed consent was obtained from the study participants and from the guardians of the pediatric subjects as per the ethical committee guidelines.

\section{Results}

A total of 22 individuals with 11 subjects in each group (vWD and hemophilia) who reported to the Hemophilia clinic during the study period were included. These individuals were age-matched with their healthy counterparts who attended the Oral Medicine clinic. The age range of the study subjects was 4-35 years. There were five females in the von Willebrand study group, and all the other study subjects were males (Table 1). 
Table 1. Distribution of the subjects according to gender and age.

\begin{tabular}{lccc}
\multicolumn{1}{c}{ Gender } & vWD Group & Hemophilia Group & Healthy Group \\
& N & N & N \\
\hline Male & 6 & 11 & 11 \\
Female & 5 & 0 & 0 \\
Mean Age (in years) & 19.45 & 19.36 & 19.45 \\
\hline
\end{tabular}

Most of the subjects were diagnosed with a congenital bleeding disorder in their early childhood. Prolonged post-traumatic bleeding from oral cavity sites like lips, tongue were found to be the initial indicators that led to the diagnosis in $36.3 \%$ of the individuals with hemophilia and $27.2 \%$ with vWD. Other causes that led to diagnosis were joint bleeds, ecchymotic patches on the skin, prolonged post-trauma bleeding from other sites and history of $\mathrm{vWD} /$ hemophilia in the family.

A total of $54.5 \%$ of the subjects with $\mathrm{vWD}$ and $63.6 \%$ of the people with hemophilia had a first or a second-degree relative who suffered from a similar bleeding diathesis. $60 \%$ of individuals with vWD had prolonged post-extraction bleeding. Prolonged bleeding following exfoliation of deciduous teeth was reported in $72.7 \%$ of the subjects with vWD and $45.4 \%$ of the hemophiliacs.

Gum bleeding was reported by more commonly reported by the vWD group (54.5\%). A percentage of $54.5 \%$ of the vWD group, $36.4 \%$ of the hemophilia group, and $27.3 \%$ of healthy subjects had never been to a dentist in the past. The brushing habit of more than once a day was reported by healthy subjects (45.5\%), followed by vWD group (36.4\%) and hemophilia group (27.3\%) (Table 2).

Table 2. Distribution of study subjects according to gum bleeding, past dental experience, brushing habit and fear regarding dental treatment.

\begin{tabular}{lccc}
\hline \multicolumn{1}{c}{ Variables } & vWD Group & Hemophilia Group & Healthy Group \\
& $\mathrm{N}(\%)$ & $\mathrm{N}(\%)$ & N $(\%)$ \\
\hline Gum Bleeding & $6(54.5)$ & $5(45.5)$ & $2(18.2)$ \\
Past Dental Visit & $5(45.5)$ & $7(63.6)$ & $8(72.7)$ \\
Excessive Post Extraction Bleeding & $3(27.3)$ & $0(0.0)$ & $0(0.0)$ \\
Brushing Habit More Than Once & $4(36.4)$ & $3(27.3)$ & $5(45.5)$ \\
Fear of Dentists / Dental Treatment & $3(27.3)$ & $2(18.2)$ & $0(0.0)$ \\
\hline
\end{tabular}

The DMFT score did not vary significantly between the groups $(\mathrm{p}=0.632)$. Higher OHI-S scores and a poorer oral hygiene status was observed more in the hemophilia group when compared to the age matched healthy subjects and vWD group.

There was no statistically significant difference in the OHI-S scores between the subjects in the three groups. More number of individuals in the von Willebrand group had good oral hygiene when compared to the subjects in the other two groups (Table 3). A total of $27.3 \%$ of vWD group, $18.2 \%$ of the hemophilia group and no subjects in the healthy group had a fear of dentists or dental treatment.

Table 3. Comparison of oral health status between the groups.

\begin{tabular}{lcccc}
\hline Status of Oral Hygiene & $\begin{array}{c}\text { vWD Group } \\
\text { Mean (SD) }\end{array}$ & $\begin{array}{c}\text { Hemophilic Group } \\
\text { Mean (SD) }\end{array}$ & $\begin{array}{c}\text { Healthy Group } \\
\text { Mean (SD) }\end{array}$ & p-value \\
\hline OHI-S Score & $1.5( \pm 1.3)$ & $2.0( \pm 1.0)$ & $1.9( \pm 1.6)$ & 0.670 \\
$\mathrm{dmft} /$ DMFT Score & $3.5( \pm 5.1)$ & $3.0( \pm 3.3)$ & $2.0( \pm 2.4)$ & 0.632 \\
\hline
\end{tabular}

\section{Discussion}


People with bleeding disorders constitute a significantly important group of the population, and there are very few studies concerning the oral aspects of the disease. To date, there are only studies that have focused on the oral health aspects in children and adolescents rather than the adult population with congenital bleeding disorders [8,9]. Hence the present study included both children and adults with bleeding disorders for the evaluation of oral hygiene and dentition status and compared them with their age-matched healthy counterparts.

The age range of the subjects in the present study ranged from 4-35 years with a mean age of 19 years. Previous authors evaluated children with von Willebrand disease and hemophilia with age range from 4 to 18 years [9], while other researchers examined subjects with type 3 Von Willebrand disease with age range of 1.6 to 17.2 years [7].

Individuals with vWD and hemophilia were mostly diagnosed during their early childhood due to prolonged bleeding episodes following minor trauma. A percentage of $45.4 \%$ of vWD, $63.6 \%$ of the hemophilia group, and $72.7 \%$ of healthy subjects had undergone dental treatment (restorations, oral prophylaxis, and dental extractions) in the past. Sixty percent of individuals with vWD had prolonged post-extraction bleeding. Other study subjects had uneventful dental experiences. Some authors reported a case of a 43-year-old patient who was diagnosed with vWD due to the prolonged post-extraction bleeding [11]. Some authors have presented a clinical case of a 30-year-old African-American male who was diagnosed with hemophilia following an episode of prolonged oral cavity bleed following dental extraction [12].

Prolonged bleeding following the shedding of deciduous teeth was reported in $72.7 \%$ of the subjects with vWD and $45.4 \%$ of the hemophiliacs. Out of this, $62.5 \%$ of vWD and $40 \%$ of the hemophiliacs had managed this bleeding episode through local measures (pressure packs, ice, tranexamic capsules) while the rest of them sought medical assistance for factor/ plasma transfusions.

Gum bleeding was seen in $54.5 \%(\mathrm{n}=6)$ of $\mathrm{vWD}, 45.5 \%(\mathrm{n}=5)$ of hemophilia group and $18.2 \%(\mathrm{n}=2)$ of the subjects in healthy group. Four subjects (36.4\%) in vWD group, $3(27.3 \%)$ in the hemophilia group, and 5 $(45.5 \%)$ in the healthy group had brushing habits more than once a day. $27.3 \%(\mathrm{n}=3)$ of $\mathrm{vWD}, 18.2 \%(\mathrm{n}=2)$ of people with hemophilia had fear of dentists/ dental treatment. The difference in these parameters was not comparable as the sample size was less in each group.

The mean dmft/DMFT scores in the vWD, hemophilia, and healthy groups were 3.5, 3, and 2, respectively. The difference in the scores in between the groups was compared, with no statistically significant differences $(p>0.05)$. There was no significant difference between the dmft scores of children with and without hemorrhagic diatheses [6]. In subjects with type 3 Von Willebrand disease, the mean DMFT score was 4, which was higher than observed in the present study [4]. However, some authors noted lower dmft/DMFT scores in the healthy subjects [5].

Most of the study subjects had a fair oral hygiene status and a predominantly good oral hygiene status was seen in the vWD group. The mean OHI-S scores in the vWD, hemophilia and healthy groups were 1.53, 2.03, and 1.90, respectively. The difference in the scores between the groups was not statistically significant $(\mathrm{p}>0.05)$. This finding was not in agreement with the findings described by other authors that observed a significant difference in the oral hygiene status between subjects with hemorrhagic diatheses and healthy subjects [6]. In a previous study, subjects with bleeding diathesis (vWD, hemophilia) had better oral hygiene status compared to healthy subjects [5]. Poor oral health status was observed in patients with type 3 vWD resulting in a greater predisposition to gingivitis and dental caries [4]. 


\section{Conclusion}

No statistically significant differences were found between the study groups. This may be due to the small sample size of the study population owing to the rarity of the occurrence of congenital bleeding disorders. However, studies involving larger a sample size are further needed to confirm the effect of bleeding disorders on oral health.

\section{Authors' Contributions}

\begin{tabular}{|c|c|c|}
\hline MK & (iD) $0000-0003-1245-2314$ & $\begin{array}{l}\text { Conceptualization, Methodology, Investigation, Formal Analysis, Writing - } \\
\text { Original Draft Preparation and Writing - Review and Editing. }\end{array}$ \\
\hline KMP & (D) $0000-0003-0967-5867$ & Methodology and Writing - Review and Editing. \\
\hline RV & (iD) $0000-0002-8932-9982$ & Methodology and Writing - Review and Editing. \\
\hline AK & (iD) $0000-0003-4986-9893$ & Writing - Review and Editing. \\
\hline
\end{tabular}

\section{Financial Support}

None.

\section{Conflict of Interest}

The authors declare no conflicts of interest.

\section{References}

[1] Sona PS, Muthu Lingam C. Hemophilia - an overview. Int J Pharm Sci Rev Res 2010; 5(1):18-26.

[2] Gupta M, Bhattacharyya M, Choudhry VP, Saxena R. Spectrum of inherited bleeding disorders in Indians. Clin Appl Thromb Hemost 2005; 11(3):325-30. https://doi.org/10.1177/107602960501100311

[3] Kumar S, Kishore R, Gupta V, Jain M, Shukla J. Prevalence and spectrum of von Willebrand disease in Eastern Uttar Pradesh. Indian J Pathol Microbiol 2010; 53(3):486-9. https://doi.org/10.4103/0377-4929.68287

[4] Stubbs M, Lloyd J. A protocol for the dental management of von Willebrand's disease, hemophilia A and hemophilia B. Aust Dent J 2001; 46(1):37-40. https://doi.org/10.1111/j.1834-7819.2001.tb00272.x

[5] van Galen KP, Engelen ET, Mauser-Bunschoten EP, van Es RJ, Schutgens RE. Antifibrinolytic therapy for preventing oral bleeding in patients with hemophilia or von Willebrand disease undergoing minor oral surgery or dental extractions. Cochrane Database Syst Rev 2015; (12):CDo11385. https://doi.org/10.1002/14651858.CD011385.pub2

[6] Kumar M, Pai KM, Kurien A, Vineetha R. Oral hygiene and dentition status in children and adults with hemophilia: a case-control study. Spec Care Dentist 2018; 38(6):391-4. https://doi.org/10.1111/scd.12324

[7] Carcao MD, Seary ME, Casas M, Winter L, Stain AM, Judd P. Dental disease in type 3 von Willebrand disease: a neglected problem. Hemophilia 2010; 16(6):943-8. https://doi.org/10.1111/j.1365-2516.2010.02344.x

[8] Nagaveni N, Arekal S, Poornima P, Hanagawady S, Yadav S. Dental health in children with congenital bleeding disorders in and around Davangere: a case-control study. J Indian Soc Pedod Prev Dent 2016; 34(1):76-81. https://doi.org/10.4103/0970-4388.175522

[9] Mielnik-Błaszczak M. Evaluation of dentition status and oral hygiene in polish children and adolescents with congenital haemorrhagic diatheses. Int J Paediatr Dent 1999; 9(2):99-103. https://doi.org/10.1046/j.1365-263x.1999.00112.x

[10] Peter S. Epidemiology etiology and prevention of dental caries. In: Peter S (Ed). Essentials of Preventive and Community Dentistry. 3rd ed. New Delhi: Arya Publishing House; 2006. pp. 222-261.

[11] Aoun N, Noujeim Z, El Toum S. Von Willebrand disease revealed after dental post-extractional bleeding: a case report. J Int Oral Health 2016; 8(4):512-7.

[12] Aledort LM, Green D, Teitel JM. Unexpected bleeding disorders. Hematology Am Soc Hematol Educ Program 2001; 1:306-21. https://doi.org/10.1182/asheducation-2001.1.306 